\title{
Treatment of Xanthelasma Palpebrarum by the Pinhole Method Using a 10,600-nm Carbon Dioxide Laser
}

Keun Jae Ahn ${ }^{1}$

Jin-Soo Kang ${ }^{2}$

Sung Bin $\mathrm{Cho}^{2}$

${ }^{1}$ Department of Science Education, Jeju National University, Jeju, Korea

${ }^{2}$ Kangskin Clinic, Seoul, Korea
Received September 17, 2013

Revised November 3, 2013

Accepted November 20, 2013

\section{Correspondence}

Sung Bin Cho

Kangskin Clinic, 1433-38, Sillim-dong,

Gwanak-gu, Seoul 151-015, Korea

Tel: +82-2-872-9007

Fax: $+82-2-872-6226$

E-mail: drsbchodgmail.com

(c) Korean Society for Laser Medicine and Surgery

(c) This is an open access article distributed under the terms of the Creative Commons Attribution NonCommercial License (http://creativecommons.org/ licenses/by-nc/3.0) which permits unrestricted noncommercial use, distribution, and reproduction in any medium, provided the original work is properly cited.
Xanthelasma palpebrarum (XP) presents clinically as yellowish plaques on the upper and/or lower eyelids. To date, several studies have reported on the therapeutic efficacies of ablative and non-ablative lasers for treatment of XP, including 10,600-nm carbon dioxide (CO2) lasers, erbium-doped yttrium aluminum garnet (YAG) lasers, 1,550-nm erbiumdoped fractional lasers, pulsed dye lasers, Q-switched neodymium: YAG lasers, and 1,450-nm diode lasers. Here, we report on a case of XP effectively treated using the pinhole method. The pinhole method utilized a CO2 laser in order to make multiple small holes, which mimicked sweat pores on skin lesions. $\mathrm{CO} 2$ lasers are among the most widely available laser devices; therefore, implementation of their use in performance of the pinhole procedure for treatment of XP in Asian patients would be relatively easy and inexpensive.

\section{Key words}

Carbon dioxide; Xanthelasma palpebrarum; Pinhole method 


\section{INTRODUCTION}

Xanthelasma palpebrarum (XP) clinically presents as yellowish plaques on the upper and/or lower eyelids. ${ }^{1}$ The histologic features of XP mainly consist of foamy histiocytes containing cholesterol crystals, lipid vacuoles, and lysosomes in the perivascular area of superficial and mid-dermis.' To date, several studies have reported on the therapeutic efficacies of ablative and nonablative lasers for XP, including 10,600-nm carbon dioxide (CO2) lasers, erbium-doped yttrium aluminum garnet (YAG) lasers, 1,550-nm erbium-doped fractional lasers, pulsed dye lasers, Q-switched neodymium (Nd):YAG lasers, and 1,450 -nm diode lasers. ${ }^{2-6}$

The pinhole method using a CO2 laser refers to a procedure in which multiple small holes mimicking sweat pores are made on skin lesions, such as burn scars and syringomas. ${ }^{7,8}$ Puncturing the targeted skin lesions with the pinhole method is easy to perform and results in less oozing and post-therapy erythema. ${ }^{7.8}$ Here, we report a case of XP effectively treated by the pinhole method.

\section{CASE REPORT}

A 63-year-old Korean woman presented with yellowish plaques on the upper and lower eyelids that had slowly progressed over the past year on the left and the past 7 years on the right. She had no pertinent family history or medical history, except for essential hypertension and hypercholesterolemia, which had been treated with oral medications for over 3 years. Upon examination, yellowish plaques were distributed across the left upper and lower eyelids and the right lower eyelid (Fig. 1A). She had no previous treatment history of eyelid lesions.

After being clinically diagnosed with XP and obtaining written informed consent, a single session of CO2 laser treatment using the pinhole method was undertaken. For local anesthesia, a topical eutectic mixture of $2.5 \%$ lidocaine hydrochloric acid and $2.5 \%$ prilocaine cream (AstraZeneca AB, Södertälje, Sweden) was applied to the lesions under occlusion one hour prior to laser treatment. The treatment parameters for the CO2 laser (SPECTRA $S P^{T M}$, Lutronic Corporation, Goyang, Koreal included a pulse duration of $200 \mu \mathrm{sec}$, a frequency of $50 \mathrm{~Hz}$, a 0.04 on-time, and a 0.01 off-time. Multiple small holes deep to the mid-dermis at intervals of 1 to $3 \mathrm{~mm}$ were made on the yellowish plaques without remarkable immediate side effects, including bleeding, oozing, or blister formation. The patient was instructed to apply $3 \%$ ofloxacin ointment (Ocuflox, Sam Il Pharm, Seoul, Korea), as well as a
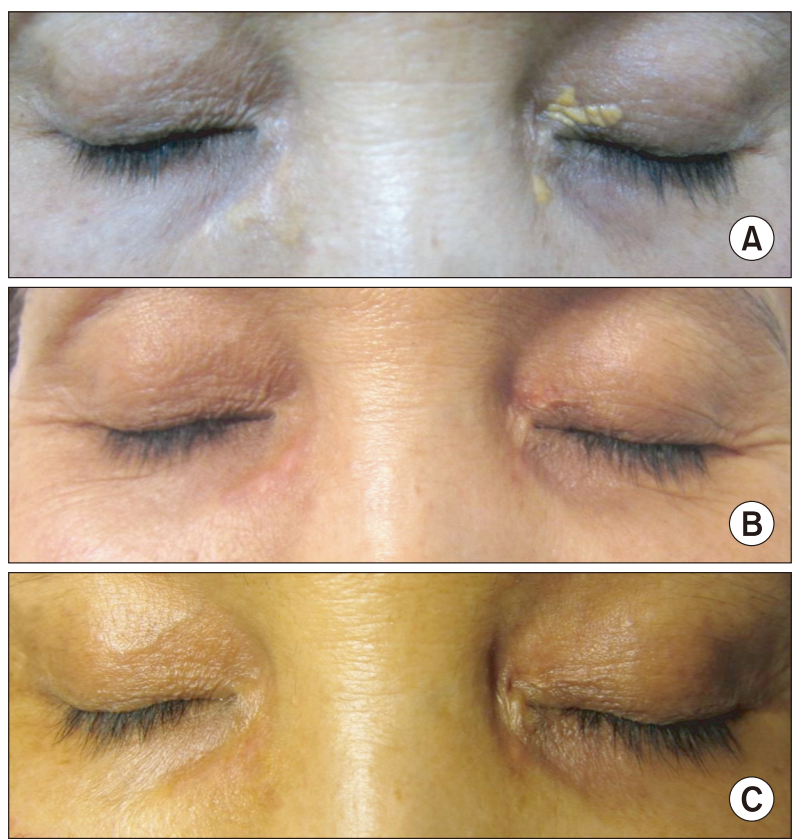

Fig. 1. (A) Yellowish plaques of xanthelasma palpebrarum (XP) on the left upper and lower eyelids and the right lower eyelid before pinhole treatment. Remarkably improved XP lesions on the eyelids at (B) one week and $(\mathrm{C})$ one month after the pinhole treatment using a 10,600-nm carbon dioxide laser.

moisturizer, to the treated area. One week after the treatment, the XP lesions were markedly improved (Fig. 1B). Post-laser therapy erythema almost disappeared within two weeks. Major side effects, including scarring, post-laser therapy dyschromia, and persistent erythema, were not observed. Most of the XP lesions were effectively treated and the patient was satisfied with the results (Fig. 1C).

\section{DISCUSSION}

Here, we report a case of XP on the upper and lower eyelids that was effectively and safely treated with the pinhole method using a CO2 laser. Multiple small holes deep to the mid-dermis at intervals of 1 to $3 \mathrm{~mm}$ were made by the pinhole method on the eyelids with XP. In Asian patients, excessive delivery of ablative C02 laser energy in an attempt to achieve better clinical outcomes can result in side effects, such as post-therapy prolonged erythema, hyper- or hypopigmentation, and scarring. However, because the epidermal architecture surrounding multiple small holes made by the pinhole method remain intact, the ablated epidermis can more rapidly recover and the risk of side effects can be reduced. ${ }^{7.8}$ 
Nonablative laser devices have been reported to be effective in the treatment of XP lesions. ${ }^{5,6}$ Previously, Park et al. ${ }^{6}$ demonstrated the use of a 1,450-nm diode laser as a new, effective, and safe therapeutic option for XP. In their report, a 1,450-nm diode laser was effectively used to destroy sebaceous glands by administering a photothermal zone to the dermis at a depth of 100-500 $\mu \mathrm{m} .{ }^{6}$ However, the penetration depths of individual laser devices are limited according to their device-specific wavelengths, and repetitive treatments are frequently required. ${ }^{2}$ Moreover, because nonablative laser devices are not widely used in many clinics, the cost-effectiveness of new laser devices for treatment of XP remains to be evaluated.

We discerned that the therapeutic efficacy of our method may be related to the following: 1) the laser induced direct and indirect thermal destruction of foamy histiocytes, and 2) the laser induced transepidermal elimination of dermal foamy contents. Laser-induced transepidermal elimination has been reported in previous studies after C02 resurfacing or nonablative 1,550-nm erbium:glass fractional laser treatment. ${ }^{9.10}$ Because only a limited portion of the XP lesions was treated with the CO2 laser in our patient, marked clinical improvement could have resulted from transepidermal elimination of the target tissues by the pinhole method.

In conclusion, we suggest the use of the pinhole method utilizing a 10,600-nm C02 laser as a new treatment modality in patients with XP on the eyelids. As CO2 lasers are some of the most widely available laser devices, their use in performing the pinhole method to treat XP in Asian patients would be relatively easy and inexpensive to implement. Additionally, the side effects associated with ablative laser procedure, especially post-therapy dyschromia and scarring in Asian skin, can be minimized. Nevertheless, further optimized, prospective studies should be conducted to confirm our findings.

\section{ACKNOWLEDGMENTS}

This research was supported by the 2013 Scientific Promotion Program funded by Jeju National University, Jeju, Korea.

\section{REFERENCES}

1. Bergman R. The pathogenesis and clinical significance of xanthelasma palpebrarum. J Am Acad Dermatol 1994;30:23642.

2. Raulin C, Schoenermark MP, Werner S, Greve B. Xanthelasma palpebrarum: treatment with the ultrapulsed CO2 laser. Lasers Surg Med 1999;24:122-7.

3. Borelli C, Kaudewitz P. Xanthelasma palpebrarum: treatment with the erbium:YAG laser. Lasers Surg Med 2001;29:260-4.

4. Katz TM, Goldberg LH, Friedman PM. Fractional photothermolysis: a new therapeutic modality for xanthelasma. Arch Dermatol 2009;145:1091-4.

5. Schönermark MP, Raulin C. Treatment of xanthelasma palpebrarum with the pulsed dye laser. Lasers Surg Med 1996; 19:336-9.

6. Park EJ, Youn SH, Cho EB, Lee GS, Hann SK, Kim KH, et al. Xanthelasma palpebrarum treatment with a 1,450-nm-diode laser. Dermatol Surg 2011;37:791-6.

7. Whang SW, Lee KY, Cho SB, Lee SJ, Kang JM, Kim YK, et al. Burn scars treated by pinhole method using a carbon dioxide laser. J Dermatol 2006;33:869-72.

8. Cho SB, Lee SH, Lee SJ, Lee JE, Kim DH. Syringoma treated with pinhole method. J Eur Acad Dermatol Venereol 2009;23: 852-3.

9. Richert SM, Bridenstine J. Transepidermal elimination of elastic fibers after carbon dioxide laser resurfacing. A report of two cases. Dermatol Surg 1998;24:275-8.

10. Wang CC, Huang CL, Lee SC, Sue YM, Leu FJ. Treatment of cosmetic tattoos with nonablative fractional laser in an animal model: a novel method with histopathologic evidence. Lasers Surg Med 2013;45:116-22. 